Original article

Paediatrics Today 2016;12(1):75-80

DOI $10.5457 / \mathrm{p} 2005-114.138$

\title{
THE EFFECT OF ANTI-SCATTER GRID ON RADIATION DOSE IN CHEST RADIOGRAPHY IN CHILDREN
}

\author{
Igor ŠABIČ ${ }^{*}$, Damjana KLJUČEVŠEK ${ }^{1}$, Martin THALER ${ }^{1}$, Dejan ŽONTAR ${ }^{2}$
}

${ }^{1}$ University Medical Center Ljubljana Children's Hospital, Radiology Unit

${ }^{2}$ Republic of Slovenia, Ministry of Health Slovenian Radiation Protection

Administration, Ljubljana, Slovenia

*Corresponding author: igor.sabic@kclj.si

Tel.: + 38615229264

Fax.: + 38615224034

Received: September 17, 2015

Accepted: January 6, 2016

Key words: Pediatrics - Image quality Chest - Digital radiography - Dose area product.

\begin{abstract}
Objective - The goal of the study was to evaluate the justification of using the anti-scatter grid in chest imaging on a digital $\mathrm{x}$-ray modality. Materials and methods - The influence of using an anti-scatter grid in chest imaging of children weighing up to $40 \mathrm{~kg}$ on image quality and patient exposure was evaluated. The technical and diagnostic quality of images was assessed according to the European guidelines „EUR16261EN« and patient exposure was determined by dose area product measurement. Additionally, image quality was graded according to subjective assessment of the evaluator. Results - The results show that imaging without the anti-scatter grid significantly reduces patient exposure. The assessment of technical image quality showed them to be comparable to even higher grades for images taken without the anti-scatter grid, while the diagnostic value significantly improved in two weight groups (the group of 10.1 to $20 \mathrm{~kg}$ and the group of $20.1 \mathrm{~kg}$ to $40 \mathrm{~kg}$ ), and a marginally significant improvement was seen in one other weight group (the group of 5.1 to $10 \mathrm{~kg}$ ). Conclusion - The results of the study indicate that the use of the anti-scatter grid in chest imaging of pediatric patients, weighting up to $40 \mathrm{~kg}$, is not justified. The images remain diagnostically adequate even after removal of the anti-scatter grid, while the patient exposure is substantially reduced.
\end{abstract}

\section{Introduction}

Digital radiology has developed rapidly over the past decade and largely replaced screenfilm radiography. The benefits of digital radiography include improvements in efficiency and image processing, as well as reduced frequency of repeated examinations (1). According to the ALARA principle, the radiation dose should be kept as low as reasonably achievable, while still providing an image quality that is adequate for an accurate diagnosis (2). The European Commission guidelines "European Guidelines on Quality Criteria for Diagnostic Radiographic Images
(EUR16261EN) « established quality criteria for diagnostic radiographic images, specifically adapted to pediatric radiology (3). Achieving such image quality with minimal doses requires not only selection of the most appropriate X-ray technology, but also optimization of the whole imaging procedure through the selection of optimal imaging parameters (4).

One of the parameters that affect the image quality is the use of the anti-scatter grid. It lies between the patient and the detector and is used to improve contrast by reducing the amount of scattered radiation that reach- 
es the detector (5). While the use of a grid improves image quality, it also increases the patient dose. However, due to the low thickness of the imaged area in infants and younger children, the use of the anti-scatter grid is often unnecessary in this population (6).

The aim of this study was to determine whether radiographic imaging of the lungs without an anti-scatter grid provides adequate diagnostic quality, and to what extent imaging without the grid reduces the dose to the patients.

\section{Material and methods}

The study included 206 children who were referred for X-ray imaging of thoracic organs as part of their regular treatment at the University Medical Center Ljubljana, Children's Hospital, Radiology Unit: 113 were male and 93 female. Their ages were between neonatal and 15 years, with an average of 3.20 years (standard deviation of 3.75 years), the median was 1.67 years and modus 0.18 years. The first quartile was 0.48 years and Q3 was 4.71 years. It was a highly asymmetrical distribution to the left, with $76.2 \%$ of all patients in the first age group (up to 5 years), $17 \%$ were aged between 5 and 10 years, $5.8 \%$ between 10 and 15 years and two patients (1\%) were older than 15 years. Since we expected that the results may depend on the weight of the patients, they were divided into 4 groups: up to $5 \mathrm{~kg}$, from 5.1 to $10 \mathrm{~kg}$, from 10.1 to 20 $\mathrm{kg}$ and more than 20 to $40 \mathrm{~kg}$. The numbers of patients in each weight group were comparable, with $43(20.9 \%)$ patients in the group of up to $5 \mathrm{~kg}, 51$ patients $(24.8 \%)$ in the group of 5.1 to $10 \mathrm{~kg}, 64(31.1 \%)$ patients in the group of 10.1 to $20 \mathrm{~kg}$, and $48(23.3 \%)$ patients were between $20.1 \mathrm{~kg}$ to $40 \mathrm{~kg}$. The results of the statistical analysis showed that there were no statistically significant differences in weight distribution between the corresponding sub-groups in any of the four weight groups.
The study was conducted in two consecutive steps. In the first, earlier step, the patients were imaged using the existing protocol that included the use of an anti-scatter grid. In the second new step, the protocol was modified so as not to include an anti-scatter gird and the next batch of patients was imaged using the modified protocol. The exposure parameters for imaging of the lung that were used in each step are summarized in Table 1. An automatic exposure control (AEC) was used for all patients. We used a (moving) antiscatter grid, with grid ratios of 8:1 and line numbers $36(1 / \mathrm{cm})$. The patients from each weight group were divided into two subgroups according to the protocol used (the original protocol with the grid or the modified protocol without it). Technical and diagnostic image quality and dose were compared between the patients of both sub-groups in each weight group.

The pediatric radiologist evaluated the technical quality of the image according to "EUR16261EN" criteria, which were evaluated with 11 elements and a final score from 1 to 3 :

1 - Unsatisfactory (very poor image and should be repeated, evaluation score 6 or less);

2 - Satisfactory (good radiogram, evaluation number 7-8);

3 - Excellent (evaluation number 9-11).

In the diagnostic part of the evaluation, the radiologist combined the technical score with 3 additional parameters: image granularity, contrast where vessels cross the ribs, and edge sharpness (based on the sharpness of the edge of the vessels in the upper lobe). Thus the diagnostic score 1 described an image with very poor quality that should be repeated, score 2 described optimum radiograms and score 3 "excellent" images, i.e. images where an accurate diagnosis could be made with lower image quality (3). The subjective evaluation score was based on the 
subjective impression of the radiologist regarding the quality of the image in relation with its diagnostic value. The scoring ranged from 1 to 4 :

1 - The image is too weak to make accurate diagnosis;

2 - The image is low quality, more details are desired, but diagnosis can be made;

3 - Good image, accurate diagnosis;

4 - Excellent image, an accurate diagnosis could be made with less detail on the image.

The study was designed as a single blind study, the entire sample being assessed by a pediatric radiologist who had no information about how individual images had been taken. The exposure of patients to ionizing radiation was determined by dose area product (DAP), measured by a built-in DAP meter (DIAMENTOR $^{\circledast}$ E2 DAP Meter, Single Channel Patient Dosemeter, PTW Freiburg GmbH, Freiburg, Germany, 2014).

\section{Ethics statement}

The study was approved by the Slovenian Medical Ethics Committee (No. 80/06/12).

\section{Statistical analysis}

For all measured parameters (technical, diagnostic and subjective image quality, and value DAP) we compared average values for the two steps in each group, compared with the nonparametric Mann-Whitney U test, because the distribution of the values of averages was not normal. IBM SPSS Statistics 20 was used and the difference was considered significant when $\mathrm{p}<0.05$.

\section{Results}

The dependence of DAP on the patient's weight is shown on Fig. 1. The linear dependence of DAP on weight can be observed for each step of the study. A clear distinction between the patients imaged with and without the grid can be observed, demonstrating the dose reduction resulting from the grid removal. It can also be seen that the effect of removing the anti-scatter grid is limited for lighter patients but increases with their weight, approaching $50 \%$ reduction at $40 \mathrm{~kg}$.

The results of the DAP measurements and image quality evaluation for both sub-groups of each weight group are listed in Table 2. In group 1 (up to $5 \mathrm{~kg}$ ) the removal of the grid led to a small (about 10\%) but still statistically significant reduction of patient dose (DAP value). In this weight group, the removal of the anti-scatter grid did not statistically significantly decrease the image quality, but even statistically significantly increased Table 1 Exposure parameters for imaging with and without an anti-scatter grid for all four weight groups

\begin{tabular}{|c|c|c|c|c|}
\hline \multirow{2}{*}{ Parameters } & \multicolumn{4}{|c|}{ Weight groups (kg) } \\
\hline & Up to 5 & 5.1 to 10 & 10.1 to 20 & 20.1 to 40 \\
\hline Anode voltage $(\mathrm{kV})$ & 102 to 109 & 102 to 109 & 109 to 117 & 117 to 125 \\
\hline Direction & $\mathrm{AP}$ & $\mathrm{AP}$ & $\mathrm{AP} / \mathrm{PA}$ & PA \\
\hline Optical density & $+2 \mathrm{OD}$ & $+2 \mathrm{OD}$ & 0 & 0 \\
\hline AEC & \multicolumn{4}{|c|}{ Yes } \\
\hline Distance & \multicolumn{4}{|c|}{$150 \mathrm{~cm}$} \\
\hline Filtration & \multicolumn{4}{|c|}{$1 \mathrm{~mm} \mathrm{Al}+0.1 \mathrm{~mm} \mathrm{Cu}$} \\
\hline Class & \multicolumn{4}{|c|}{ S400 } \\
\hline Focus & \multicolumn{4}{|c|}{ Large $(1 \mathrm{~mm})$} \\
\hline
\end{tabular}

$\mathrm{AP}=$ Anterior-posterior; $\mathrm{PA}=$ Posterior-anterior; $\mathrm{AEC}=$ Automatic exposure control. 


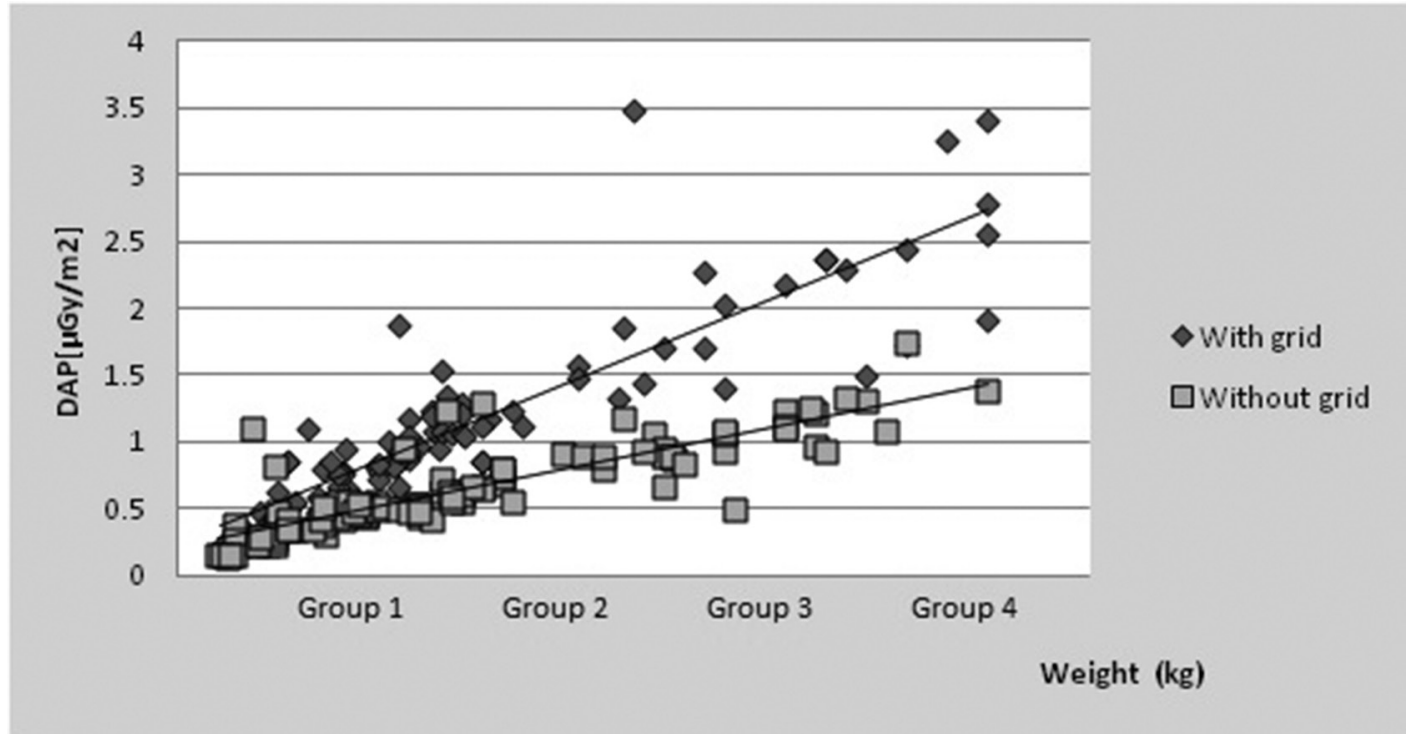

Fig. 1 Dependence between DAP and weight, by anti-scatter grid use. Group $1=u p$ to $5 \mathrm{~kg}$, Group 2=from 5.1 to $10 \mathrm{~kg}$, Group 3=from 10.1 to $20 \mathrm{~kg}$, Group $4=20.1$ to $40 \mathrm{~kg}$.

the average grade of subjective assessment. In group $2(5.1$ to $10 \mathrm{~kg}$ ) the DAP value statistically significantly decreased after the removal of the grid, by approximately $40 \%$. The image quality for technical, diagnostic and subjective assessment remained the same or was even slightly improved. In group 3 (10.1 to $20 \mathrm{~kg}$ ) removal of the grid led to a decrease in the average DAP value of approximately $40 \%$. The results show that the difference in the technical, diagnostic and subjective image quality evaluation is statistically signifi-

Table 2 Results of the DAP measurements and image quality assessment for groups up to $5 \mathrm{~kg}, 5.1$ to 10 $\mathrm{kg}, 10.1$ to $20 \mathrm{~kg}$ and 20.1 to $40 \mathrm{~kg}$. The values listed represent the average value \pm one standard deviation

\begin{tabular}{|c|c|c|c|c|c|c|c|c|}
\hline \multirow{3}{*}{$\begin{array}{l}\text { Parameters } \\
\mathrm{n}\end{array}$} & \multicolumn{8}{|c|}{ Weight groups (kg) } \\
\hline & \multicolumn{2}{|l|}{ up to 5} & \multicolumn{2}{|l|}{5.1 to 10} & \multicolumn{2}{|l|}{10.1 to 20} & \multicolumn{2}{|l|}{20.1 to 40} \\
\hline & 16 & 27 & 21 & 30 & 34 & 30 & 21 & 27 \\
\hline Grid & Yes & No & Yes & No & Yes & No & Yes & No \\
\hline Weight (kg; $\bar{\chi} \pm S D)$ & $3.56 \pm 0.83$ & $3.74 \pm 1.0$ & $7.72 \pm 1.44$ & $8.03 \pm 1.32$ & $13.30 \pm 2.39$ & $13.89 \pm 2.28$ & $30.97 \pm 6,6$ & $28,12 \pm 4,98$ \\
\hline $\mathrm{p}^{*}$ & \multicolumn{2}{|l|}{$\mathrm{p}=0.642$} & \multicolumn{2}{|l|}{$\mathrm{p}=0.455$} & \multicolumn{2}{|l|}{$\mathrm{p}=0.189$} & \multicolumn{2}{|l|}{$\mathrm{p}=0.148$} \\
\hline $\mathrm{DAP}(\mu \mathrm{Gym} 2 ; \bar{\chi} \pm \mathrm{SD})$ & $0.33 \pm 0.11$ & $0.30 \pm 0.21$ & $0.75 \pm 0.15$ & $0.46 \pm 0.07$ & $1.11 \pm 0.25$ & $0.67 \pm 0.21$ & $2.19 \pm 0,64$ & $1.04 \pm 0.25$ \\
\hline$\% ; p^{*}$ & \multicolumn{2}{|c|}{$10 \%(\mathrm{p}=0.040)$} & \multicolumn{2}{|c|}{$40 \%(\mathrm{p}<10-3)$} & \multicolumn{2}{|c|}{$40 \%(\mathrm{p}<10-3)$} & \multicolumn{2}{|c|}{$50 \%(\mathrm{p}<10-3)$} \\
\hline $\operatorname{TE}(1-3 ; \bar{\chi} \pm \mathrm{SD})$ & $2.55 \pm 0.21$ & $2.66 \pm 0.18$ & $2.62 \pm 012$ & $2.73 \pm 0.17$ & $2.51 \pm 0,27$ & $2.74 \pm 0,14$ & $2.68 \pm 0.23$ & $2.81 \pm 0,17$ \\
\hline$\% ; \mathrm{p}^{*}$ & \multicolumn{2}{|c|}{$4 \%(\mathrm{p}=0.131)$} & \multicolumn{2}{|c|}{$4 \%(\mathrm{p}=0.011)$} & \multicolumn{2}{|c|}{$8 \%(\mathrm{p}=0.001)$} & \multicolumn{2}{|c|}{$4 \%(\mathrm{p}=0.057)$} \\
\hline $\mathrm{DE}(1-3 ; \bar{\chi} \pm \mathrm{SD})$ & $2.72 \pm 0.30$ & $2.80 \pm 0.26$ & $2.76 \pm 0.23$ & $2.87 \pm 0.20$ & $2.52 \pm 0.37$ & $2.83 \pm 0.22$ & $2.67 \pm 0.37$ & $2.93 \pm 0.12$ \\
\hline$\% ; p^{*}$ & \multicolumn{2}{|c|}{$3 \%(\mathrm{p}=0.376)$} & \multicolumn{2}{|c|}{$3 \%(\mathrm{p}=0.088)$} & \multicolumn{2}{|c|}{$10 \%(\mathrm{p}=0.001)$} & \multicolumn{2}{|c|}{$9 \%(\mathrm{p}=0.003)$} \\
\hline $\mathrm{SE}(1-4 ; \bar{\chi} \pm \mathrm{SD})$ & $2.75 \pm 0.68$ & $3.51 \pm 0.51$ & $2.95 \pm 0.22$ & $3.47 \pm 0.51$ & $2.62 \pm 0.55$ & $3.40 \pm 0.50$ & $2.81 \pm 0.40$ & $3.15 \pm 0.36$ \\
\hline$\% ; p^{*}$ & \multicolumn{2}{|c|}{$19 \%(\mathrm{p}<10-3)$} & \multicolumn{2}{|c|}{$13 \%(\mathrm{p}<10-3)$} & \multicolumn{2}{|c|}{$20 \%(\mathrm{p}<10-3)$} & \multicolumn{2}{|c|}{$8 \%(\mathrm{p}=0.005)$} \\
\hline
\end{tabular}

$\mathrm{DAP}=$ dose area product; $\mathrm{TE}=$ Technical evaluation; $\mathrm{DE}=$ Diagnostic evaluation; $\mathrm{SE}=$ Subjective evaluation. ${ }^{*} \mathrm{p}=\mathrm{Difference}$ between imaging with and without grid calculated as (score with grid - score without grid)/score with grid). 
cant. In group 4 (20.1 to $40 \mathrm{~kg}$ ) the DAP value decreased by approximately $50 \%$, again a statistically significant difference. The difference in the technical grades is not statistically significant, while the differences in the grades of diagnostic and subjective assessment are statistically significant.

\section{Discussion}

The results of the study show that in all four weight groups evaluated patient doses (DAP) were significantly reduced by removing the grid. The actual dose reduction increased with patient weight, and varied from about $10 \%$ in the group of patients up to $5 \mathrm{~kg}$ to about $50 \%$ in group of 20.1 to $40 \mathrm{~kg}$. As the weight of the patient affects the value of DAP, the results of the grid influence on the patient dose could be affected by differences in patient weight distributions between the two sub-groups belonging to each weight group. A statistical comparison of the distributions of patient weights in each sub-group was thus performed to check for this potential source of bias. The results of other studies also report a reduction in dose after removal of the grid, while the level of reduction varies considerably from study to study (7).

The results of our study show that removing the grid did not reduce the image quality. In fact the image quality not only remained diagnostically appropriate, but in some cases the quality of the images taken without a grid received even better grades than those taken with a grid. This statement is contrary to the findings of the reviewed literature that report a significant decline in image quality after removal of the grid (1, 8-9). This discrepancy may be due to the fact that we focused on the diagnostic value of the images rather than on the physical parameters of image quality, such as contrast and signal-to-noise ratio. Since the physical parameters of image quality do not necessarily reflect its diagnostic value, it is possible that the decline in image quality, in terms of physical parameters, did not affect its diagnostic value. Another relevant factor may be the detector technology used in the study. As reported previously, the improved technical characteristics of modern digital detectors may make them less affected by the removal of the anti-scatter grid than was the case with conventional technologies used in older studies $(9,10-11)$. One of the main limitations of this study is that the images were graded by a single evaluator. Therefore, further studies would be required to validate the reported improvement.

\section{Conclusion}

The research demonstrated that the appropriate image quality and diagnostic value of chest radiogram may be achieved even without an anti-scatter grid. At the same time, the removal of the grid leads to a significant reduction in the patient's exposure to ionizing radiation. Based on the results presented, we may conclude that the use of anti-scatter grids in chest imaging of pediatric patients weighing up to $40 \mathrm{~kg}$ is not justified. The radiological section has already introduced changes to protocols for imaging this group of patients, so the anti-scatter grid is no longer in use.

Acknowledgement: The study was approved by the Republic of Slovenia National Medical Ethics Committee (KME / NMEC), number 80/06/12, as of 29. 6. 2012 .

Authors' contributions: Conception and design: IS, DZ, DK; Acquisition, analysis and interpretation of data: IS, DZ, MT; Drafting the article: IS, DK; Revising it critically for important intellectual content: DZ, DK.

Conflict of interest: The authors declare that they have no conflict of interest.

\section{References}

1. Sanchez JR, Vano-Galvan E, Vano E, Ruiz GN, Fernandez Soto JM, Martinez Barrio D, et al. 
Optimising the use of Computed Radiography in Pediatric Chest Imaging. J Digit Imaging. 2009;22(2):104-13.

2. Kiljunen T1, Tietäväinen A, Parviainen T, Viitala A, Kortesniemi M. Organ doses and effective doses in pediatric radiography: patient-dose survey in Finland. Acta Radiol. 2009;50(1):114-24.

3. European Commission. European Guidelines on Quality Criteria for Diagnostic Radiographic Images in Paediatrics. Luxembourg: Office for Official Publications of the European Communities; 1996. EUR 16261 EN.

4. Chapple CL. Optimisation of Protection in Paediatric Radiology. Westgate Rd, Newcastle General Hospital. Regional Medical Physics Department. 2000. Available: http://irpa12.org.ar/PDF/RC/ RC-14_fullpaper.pdf.

5. Minigh J. Pediatric Radiation Protection. Radiol Technol. 2005;76(5):365-75.

6. Gould R, Doyle P, McFadden S, Hughes C. Potential Dose Optimisation in Paediatric Digital Ra- diography. The UK Radiological Congress-Medical Imaging. 2014;1(1):1 pp.

7. Fritz S, Jones AK. Guidelines for Anti-scatter Grid Use in Pediatric Digital Radiography. Pediatr Radiol. 2014;44(3):313-21.

8. Ween B, Olstad M, Jakobsen JA, Olsen DR. Pediatric Digital Chest Radiography, Comparison of Grid versus Non-grid Techniques. Eur Journal of Radiography. 2009;1(4):201-6.

9. King JM, Idris A Elbakri IA, Reed M. Antiscatter Grid use in Pediatric Digital Tomosynthesis Imaging. Journal of Applied Clinical Medical Physics. 2011;12(4):221-30.

10. Neitzel U. Management of Pediatric Radiation Dose using Philips Digital Radiography. Pediatr Radiol. 2004;34(Suppl 3):227-33.

11. McDonald S, Martin CJ, Darragh CL, Graham DT. Dose-area product measurements in paediatric radiography. Br J Radiol. 1996;69(820):318-25. 